\title{
STEREO and ACE Observations of Energetic Particles from Corotating Interaction Regions
}

\author{
R. A. Leske*, R. A. Mewaldt*, G. M. Mason ${ }^{\dagger}$, C. M. S. Cohen*, A. C. Cummings*, \\ A. W. Labrador*, E. C. Stone*, M. E. Wiedenbeck ${ }^{* *}$ and T. T. von Rosenvinge ${ }^{\ddagger}$ \\ ${ }^{*}$ California Institute of Technology, Pasadena, CA 91125 USA \\ ${ }^{\dagger}$ Johns Hopkins University/Applied Physics Laboratory, Laurel, MD 20723 USA \\ ** Jet Propulsion Laboratory, California Institute of Technology, Pasadena, CA 91109 USA \\ ${ }^{\ddagger}$ NASA/Goddard Space Flight Center, Greenbelt, MD 20771 USA
}

\begin{abstract}
Since early 2007, significant particle enhancements due to corotating interaction regions (CIRs) have regularly appeared at 1 AU without any appreciable contamination from solar energetic particles (SEPs). In 2009 the prevalence of CIRs diminished as the maximum speed of the high speed solar wind streams in the ecliptic decreased along with the tilt of the heliospheric current sheet. Observations of CIR time profiles at different longitudes from STEREO show delays between the Behind and Ahead spacecraft that are often roughly as expected from the corotation time lag, although small differences in the spacecraft latitudes introduce significant scatter in the time delays. In some cases different features seen at Ahead and Behind suggest that transient disturbances in the solar wind may alter connection to or transport from the shock, or that temporal changes occur in the CIR shock itself. $\mathrm{H}$ and He data from STEREO/LET at 1.8-6 MeV/nucleon show that 1) the CIR spectral index at these energies is $\sim-4$, independent of intensity but with considerable variability, 2) the $\mathrm{He} / \mathrm{H}$ ratio is $\sim 0.03$ for larger CIRs but varies systematically with energy and event intensity, and 3) although the correlation between the $\mathrm{CIR} \mathrm{MeV}$ particle increases and solar wind speed is generally good, many times a high-speed stream is not associated with $\mathrm{MeV}$ particles, while at other times a recurring series of CIR particle increases appears only at higher energies and may be associated with current sheet crossings and low speed solar wind.
\end{abstract}

Keywords: Interplanetary physics, Corotating streams, Energetic particles, STEREO, ACE

PACS: $96.50 .-\mathrm{e}, 96.50 . \mathrm{Qx}, 96.50 . \mathrm{Vg}$

\section{INTRODUCTION}

Corotating interaction regions (CIRs) form when a highspeed solar wind stream overtakes and interacts with a slower-speed stream. Shocks may develop at CIR boundaries, usually beyond $1 \mathrm{AU}$, and may accelerate particles to $\sim 20 \mathrm{MeV} /$ nucleon that diffuse inward along the magnetic field to $1 \mathrm{AU}$ [1]. CIRs are especially prominent during the declining phase of the solar cycle, when the heliospheric magnetic field has a well-developed sector structure and coronal holes may extend to low latitudes. Since early 2007 frequent CIR particle enhancements have appeared at $1 \mathrm{AU}$, practically free of solar energetic particle (SEP) contamination during the present long, deep solar minimum. During this period, the twin STEREO spacecraft have been making in-situ observations from locations up to $\sim 110^{\circ}$ apart in longitude and up to $\sim 11^{\circ}$ apart in heliographic latitude near 1 $\mathrm{AU}[2,3]$. Included in the IMPACT instrument suite on STEREO is the Low Energy Telescope (LET) [4] which measures elemental composition from $\mathrm{H}$ to beyond $\mathrm{Ni}$ at $\sim 2$ to $\sim 50 \mathrm{MeV} /$ nucleon. Here we report updated CIR observations from LET during the present solar minimum; earlier results from this study may be found in $[2,3]$.

\section{OBSERVATIONS}

An overview of the recent CIR energetic particle activity is shown in Fig. 1. Except for a few small SEP events as indicated, nearly all the low energy protons detected by STEREO/LET since early 2007 have been due to CIRs. Note that many high-speed streams are not associated with particle activity at $1 \mathrm{AU}$ at $\mathrm{MeV}$ energies. Differences in whether the streams create a shock, the characteristics of any such shocks (location, size, strength, obliquity, etc.), and connection of the observer to the shock presumably account for the differences in the particle activity. In 2009 the prevalence of the high-speed streams and their maximum speed has decreased, and along with it the occurrence rate of CIRs. This appears to correspond to a decrease in the tilt of the heliospheric current sheet below $\sim 30^{\circ}[5]$ and is typical of CIR behavior at solar minimum.

Comparisons of the CIR time profiles observed at the two STEREO spacecraft, Ahead and Behind, are discussed in some detail in $[2,6]$. The timing differences in CIR features observed at the two spacecraft have grown to $\sim 8$ days, roughly following the trend we calculate for corotation (accounting for the changing radial and longitudinal separation of the spacecraft). The expected 


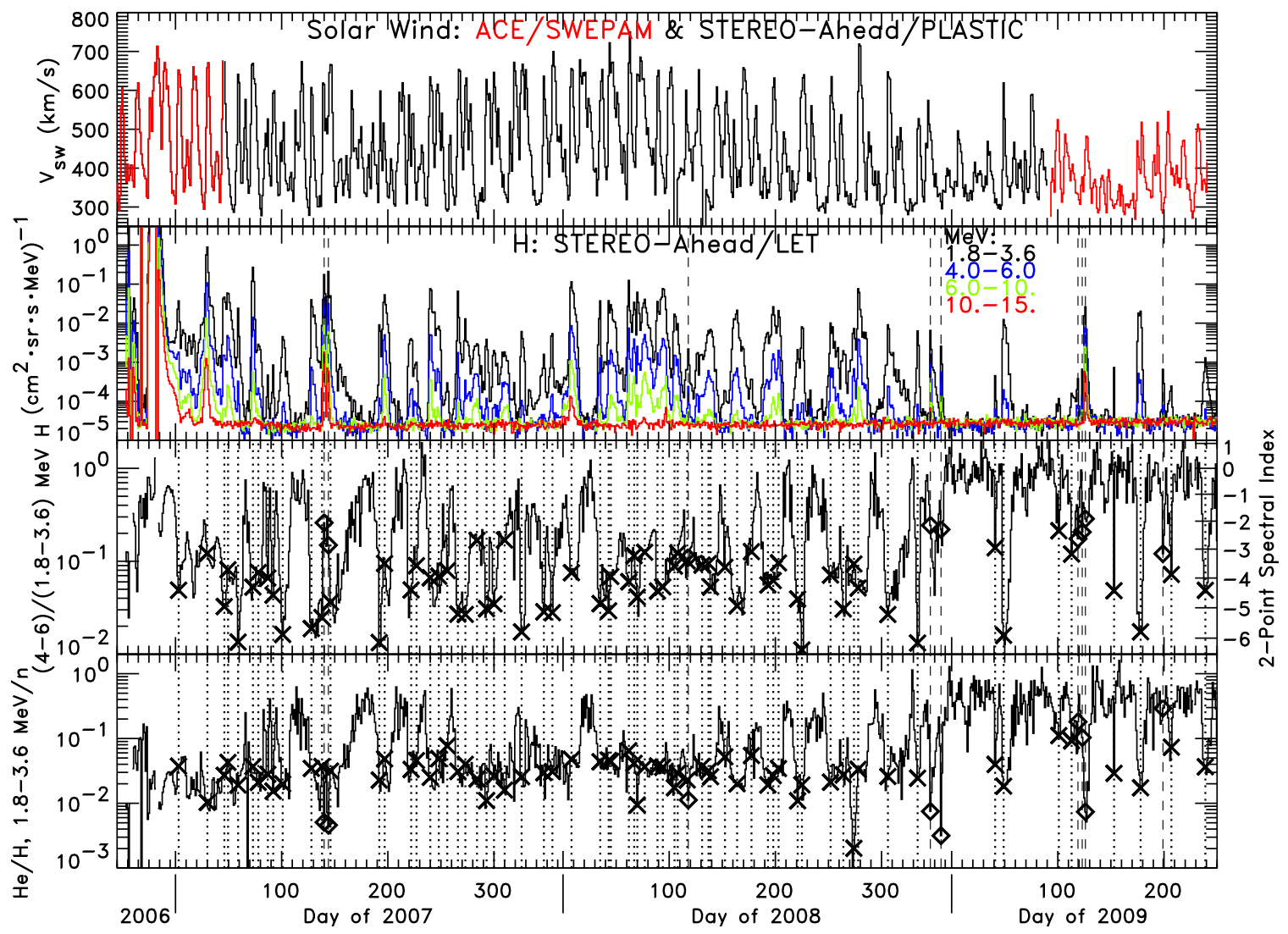

FIGURE 1. Daily-averaged solar wind speeds (top panel) from PLASTIC on STEREO-Ahead (black) or SWEPAM on ACE (red, when PLASTIC data are unavailable), compared with daily-averaged proton intensities from STEREO/LET at the energies indicated (second panel). The third panel shows the ratio of the (4-6)/(1.8-3.6) MeV proton intensities, with the corresponding power-law spectral index shown on the right axis; the bottom panel shows the $\mathrm{He} / \mathrm{H}$ ratio at 1.8-3.6 MeV/nucleon. Dotted lines mark the local maxima of CIR events; dashed lines indicate likely small SEP events. Values of the ratios in the bottom two panels at the CIR peaks and at SEP peaks are indicated by crosses and diamonds, respectively.

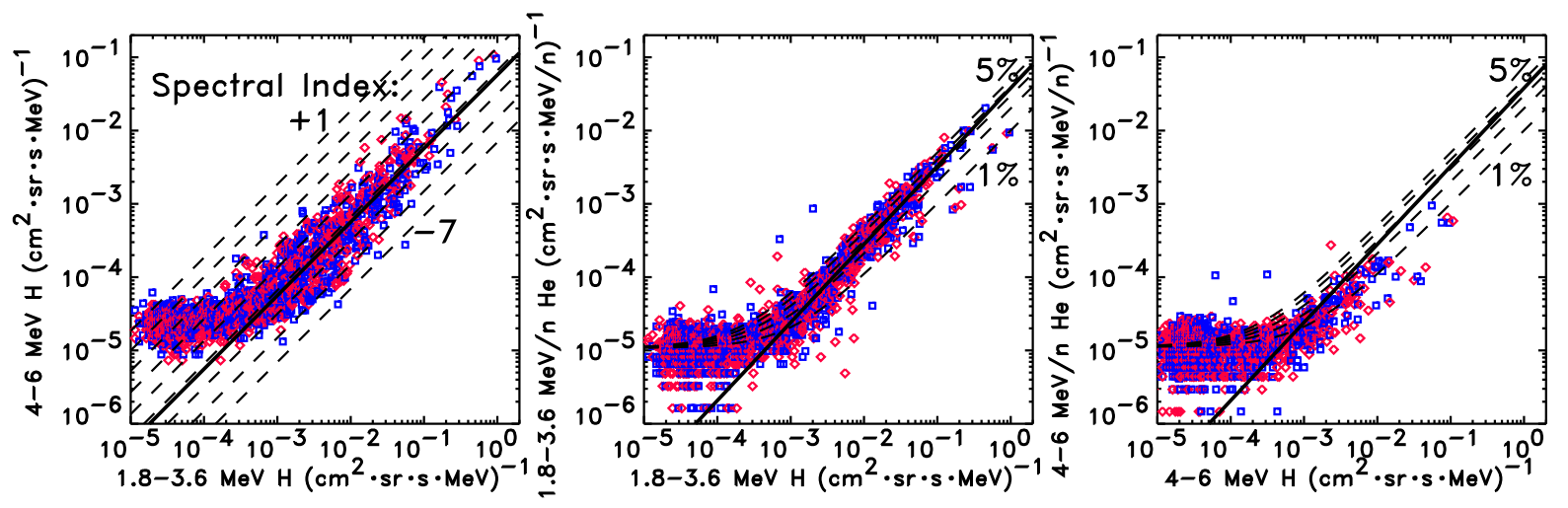

FIGURE 2. Daily-averaged $\mathrm{H}$ and He intensities (at the indicated energies) from LET on STEREO-Ahead (red diamonds) and Behind (blue squares) from the start of 2007 to day 245 of 2009. Dashed lines in the first panel indicate spectral indices of +1 to -7 , while in the last two panels $\mathrm{He} / \mathrm{H}$ ratios of $5 \%$ to $1 \%$ (assuming a minimum He intensity of $10^{-5}$ ) are indicated. The thick solid lines in the first two panels are fits to the data for $1.8-3.6 \mathrm{MeV} \mathrm{H}$ intensities above $10^{-3}$. The fit from the second panel has been copied into the last panel to illustrate that the higher energy data fall below this fit and thus have a lower $\mathrm{He} / \mathrm{H}$ ratio. Note that the fit is parallel to the constant index lines in the first panel but not parallel to the constant $\mathrm{He} / \mathrm{H}$ lines in the other panels; see text. 
spread in the timing differences due to different solar wind speeds is less than $\sim 0.5$ days whereas the scatter in the observed timing differences is often several days. However, the heliographic latitude of the two spacecraft can differ by several degrees, even though both orbit in the ecliptic. If the CIR boundaries have a low inclination to the heliographic equator, a small difference in spacecraft latitudes can easily introduce a timing spread of the observed magnitude [2].

Peak CIR event intensities observed by the STEREO and ACE spacecraft sometimes differ systematically, either due to temporal changes in the intensities over the several-day interval between observations or due to spatial (radial or latitudinal) differences between the observers [3]. In cases where the radial separation between the observers is small, it seems likely that a major contributor to the intensity differences is a latitudinal gradient [3]; a similar conclusion was independently reached for other STEREO events at lower energies $[6,7]$.

Latitudinal effects alone do not account for all differences between the observers. Around days 70 and 95 of 2008, particle increases appeared only at Ahead, not Behind or ACE, although all three spacecraft were at essentially the same latitude [3] and there was no obvious evidence of any associated flare activity on the Sun. A disturbance in the solar wind may have altered connection to a CIR shock or transport from it, but a recurrence one rotation later seems unlikely. Additional study of CIRs from multiple vantage points may help to better explain such observations.

The CIR H spectra (Fig. 1) at several MeV are soft and rather variable, with indices ranging from $\sim-3$ to $\sim-6$, while $\mathrm{He} / \mathrm{H}$ varies by a factor of $\sim 2$ on either side of $\sim 0.03$. Both quantities are similar to values reported in other studies at these energies $[1,8,9]$. The small SEP events in Fig. 1 typically had harder spectra but lower $\mathrm{He} / \mathrm{H}$ ratios, while the hardest spectra and highest $\mathrm{He} / \mathrm{H}$ values appear at the lowest $\mathrm{H}$ intensities and are due to quiet-time anomalous and galactic cosmic rays.

As shown by the fit in the left panel of Fig. 2, the average $H$ spectral index is independent of the intensity, as has also been observed for $\sim 400 \mathrm{keV} /$ nucleon CIR He [7]. However, the middle panel of Fig. 2 indicates that while the CIR $\mathrm{He} / \mathrm{H}$ ratio at $1.8-3.6 \mathrm{MeV} /$ nucleon is typically $\sim 0.03$, it is larger at high intensities and smaller at lower intensities. (Although quiet-time background is present, correcting for it would only reduce the CIR $\mathrm{He} / \mathrm{H}$ ratio at low intensities even more, accentuating the effect.) At slightly higher energies (right panel of Fig. 2), the $\mathrm{He} / \mathrm{H}$ ratio is significantly less, confirming the energy dependence we observed earlier in a small sample of events [2] and qualitatively consistent with $\mathrm{H}$ and $\mathrm{He}$ spectral behavior reported by [10]. This is curious since CIR heavier ion abundance ratios are nearly independent of energy over this same energy range [8].

\section{DISCUSSION}

The dependence on energy and event intensity in the $\mathrm{He} / \mathrm{H}$ ratio (Fig. 2) suggests that the He spectrum generally rolls over, or breaks, at lower energies than $\mathrm{H}$, and that this break energy may be higher for larger events; both these effects are often seen in SEP spectra. However, one might then expect the break energy of $\mathrm{H}$ to also depend on the event size, in which case measurements in a fixed energy band would show a dependence of the $\mathrm{H}$ spectral index on intensity, which is not observed.

A correlation between the speed of the solar wind streams and the CIR He/H ratio has been reported in earlier studies [1, 9]. Together with the fact that the $\mathrm{He} / \mathrm{H}$ ratio is typically higher in the fast solar wind than in the slow wind, the observations might indicate that the higher-speed streams generate and serve as seed material for the more-intense CIR events. When measurements of solar wind $\mathrm{He} / \mathrm{H}$ ratios from PLASTIC become available, we will be able to directly test the association between CIR and solar wind composition, similar to what was done for $\mathrm{Fe} / \mathrm{O}$ at lower energies in [8].

The recurring nature of the CIRs observed in this study is evident in Fig. 3. Here a strip color-coded by the LET 1.8-3.6 MeV $\mathrm{H}$ intensity has been plotted versus time, after being wrapped to show the phase within a solar rotation (more specifically, the day of 2007 modulo 27). Events recurring one solar rotation apart form nearly horizontal bands on this plot. For comparison, the time periods of CIRs in the lower-energy (320-450 keV/nucleon) survey of Mason et al. [7] are indicated. Curiously, in addition to the bands across the top and bottom of the plot, there is a prominent, nearly vertical streak (circled), which would correspond to a corotation period $\sim 10 \%$ longer than those of the more nearly horizontal bands. Although obvious at $\mathrm{MeV}$ energies, none of the lower energy CIRs appear on the vertical streak, and we also find a poorer correlation of CIR particle events with the solar wind speed during this period. These events are definitely CIRs and not SEPs. In addition to their multiple recurrences, their spectral hardness and $\mathrm{He} / \mathrm{H}$ ratios agree with those of the other, typical CIRs and they are present in both Ahead and Behind with a time lag between Behind and Ahead reasonable for CIRs.

Time profiles of selected CIRs in LET protons and in lower energy protons from the Suprathermal Ion Telescope (SIT) on STEREO [11] are shown in Fig. 4 along with the solar wind speed from STEREO/PLASTIC and the magnetic field longitude from STEREO/MAG. The left column shows a typical event from the upper horizontal band in Fig. 3. The profiles in LET and SIT appear similar in this event and peak near the time of maximum solar wind speed, shortly after a clear magnetic sector crossing. The middle and right columns of Fig. 4 show examples from the nearly vertical streak in Fig. 3 


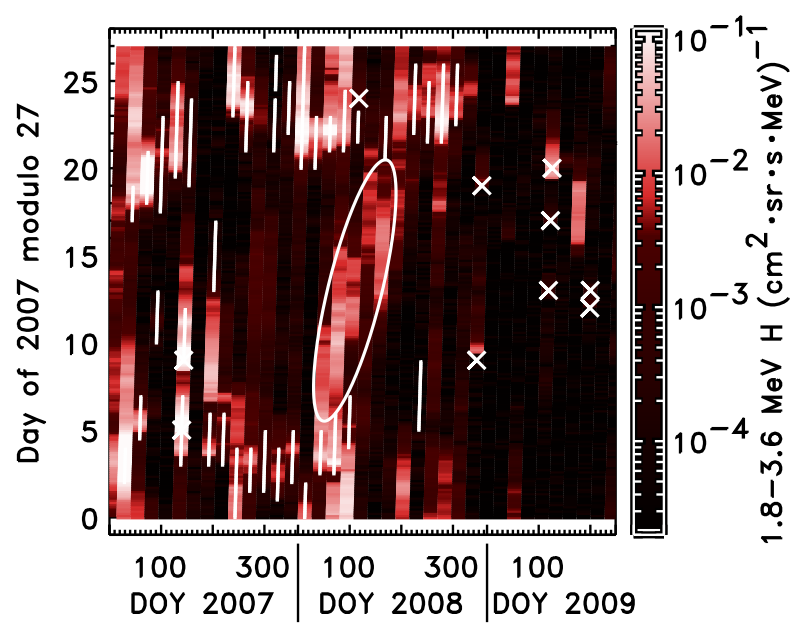

FIGURE 3. Intensity of 1.8-3.6 MeV H from LET/Ahead (color scale) plotted vs time, modulo 27 days. White crosses mark likely SEP events; white lines are CIR periods from [7]. The circled region is discussed in the text.

(data from STEREO-Behind are used in the third column due to a data gap in PLASTIC on Ahead during this event). The SIT $0.39 \mathrm{MeV}$ proton intensities are suppressed relative to LET $1.8-3.6 \mathrm{MeV}$ protons by nearly two orders of magnitude in the third column (and in the second of two LET enhancements in the second column) compared to the first. Timing of the event in LET relative to the solar wind speed may also be shifted; note that for the third column the event peaks in LET during the slow speed $(<400 \mathrm{~km} / \mathrm{sec})$ solar wind. The magnetic longitude shows multiple, brief excursions to the opposite sector, suggesting that the spacecraft may be skimming along the heliospheric current sheet. Unusual CIR events with similar time profiles, spectra, and correlation with plasma parameters were reported in [12].

Perhaps a distant interaction region is located near the current sheet, and particles reach the spacecraft via drift along the sheet rather than by travel along the magnetic field line, similar to what might be concluded from [13]. Suppression of the low energy particles in these events might be due differences in the diffusion coefficient, transport over a larger distance, or preferential transport of the higher energy particles along the current sheet [14]. These unusual events will certainly be the subject of continued study, and together with detailed modeling, may help to further elucidate the physics of particle acceleration and transport in the heliosphere.

\section{ACKNOWLEDGMENTS}

This work was supported by NASA at Caltech and JPL under sub-contract SA2715-26309 from UC Berke-
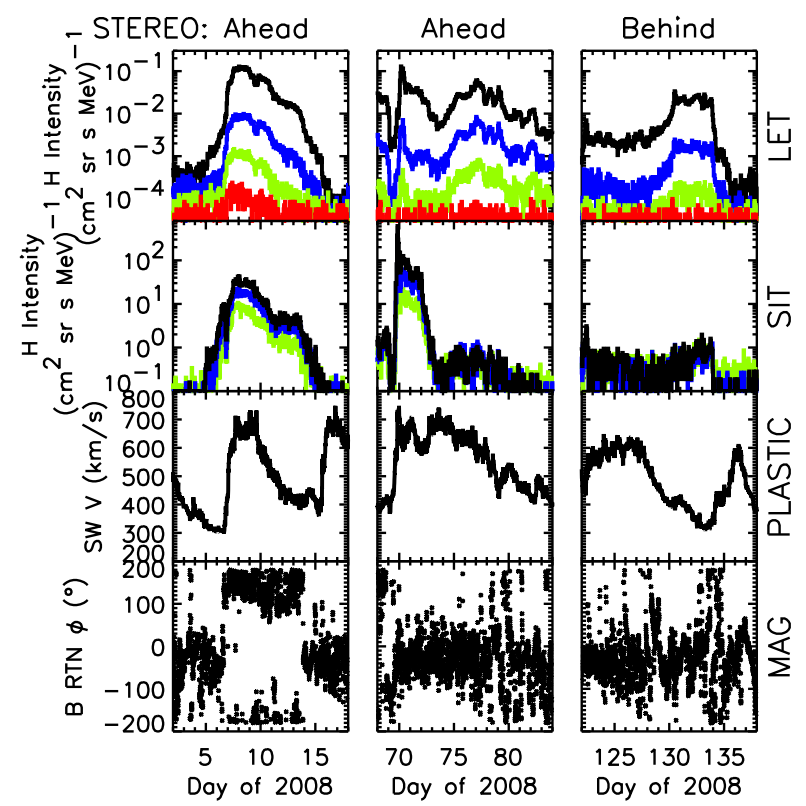

FIGURE 4. Hourly-averaged intensities of $\mathrm{H}$ from STEREO/LET at 1.8-15 MeV as in Fig. 1 (top panels) and from STEREO/SIT at 0.39, 0.55, and $0.78 \mathrm{MeV}$ (black, blue, and green, respectively; second panels from top), compared with hourly-averaged solar wind speed from STEREO/PLASTIC (third panels from top) and 10-minute averaged magnetic field longitude from STEREO/MAG (bottom panels).

ley under NASA contract NAS5-03131. We thank STEREO/PLASTIC (NASA contract NAS5-00132), STEREO/MAG, and ACE/SWEPAM investigators for making their data publicly available.

\section{REFERENCES}

1. F. E. Marshall and E. C. Stone, JGR 83, 3289 (1978).

2. R. A. Leske, et al., in Particle Acceleration and Transport in the Heliosphere and Beyond, eds. G. Li, et al., AIP Conf. Proc. 1039 (Melville, NY: AIP), 131 (2008).

3. R. A. Leske, et al., Proc. 31st ICRC (Łódź), SH 2.3, paper 0537 (2009).

4. R. A. Mewaldt, et al., Space Sci. Rev. 136, 285 (2007).

5. The Wilcox Solar Observatory website. URL: http://wso.stanford.edu/

6. R. Gómez-Herrero, et al., JGR 114, A05101 (2009).

7. G. M. Mason, et al., Sol. Phys. 256, 393, (2009).

8. G. M. Mason, et al., ApJ 678, 1458 (2008).

9. I. G. Richardson, et al., JGR 98, 13 (1993).

10. R. A. Mewaldt, E. C. Stone, and R. E. Vogt, GRL 6, 589 (1979).

11. G. M. Mason, et al., Space Sci. Rev. 136, 257 (2007).

12. D. V. Reames, et al., GRL 24, 2917 (1997).

13. T. R. Sanderson, et al., GRL 26, 1785 (1999).

14. J. R. Jokipii, E. H. Levy, and W. B. Hubbard, ApJ 213, 861 (1977). 